\title{
OPTIMAL FUNCTIONS FOR A PERIODIC UNCERTAINTY PRINCIPLE AND MULTIRESOLUTION ANALYSIS*
}

\author{
by JÜRGEN PRESTIN and EWALD QUAK
}

(Received 20th April 1996)

Dedicated to Professor Lothar Berg

on the occasion of his 65 th birthday

\begin{abstract}
In this paper, it is shown that certain Theta functions are asymptotically optimal for the periodic time frequency uncertainty principle described by Breitenberger in [3]. These extremal functions give rise to a periodic multiresolution analysis where the corresponding wavelets also show similar localization properties.
\end{abstract}

1991 Mathematics subject classification: Primary 42A16, Secondary 26D05, 26D10, $26 \mathrm{D} 15$.

\section{Introduction}

A fundamental result on time and frequency localization of square-integrable functions on the real line is given by the Heisenberg uncertainty principle, and it is well known that the Gaussian functions serve as extremal functions for this inequality. On the other hand, uncertainty relations for periodic functions have not been studied as thoroughly as the original inequality on the real axis. In this paper, we want to focus on a concept discussed by Breitenberger in [3], where uncertainty of a periodic squareintegrable function is described in terms of the product of frequency and angular variances. For more detailed information concerning this particular periodic uncertainty principle, we refer the interested reader to Breitenberger's paper [3] and the references therein. Further related topics in time-frequency localization in connection to multiresolution are treated in Benedetto, Heil and Walnut [1], Chui-Wang [4-6], Daubechies [8] and Cohen [7].

A unified approach to the theory of general periodic multiresolution analysis is investigated by Plonka and Tasche [13], Koh, Lee and Tan [9], Narcowich and Ward [10]. The time frequency localization of periodic scaling functions and wavelets according to the aforementioned uncertainty relation is studied intensively by Narcowich and Ward. The periodic basis functions in [10] possess an uncertainty product of $\mathcal{O}(\sqrt{n})$ for increasing dimension $n$ of the corresponding spaces. Uniformly bounded uncertainty products are computed by Selig [16] for trigonometric

- Research of the authors supported by NATO CRG Programme, Grant No 950681. 
fundamental Lagrange interpolants based on de la Vallée Poussin means and for trigonometric Hermite interpolants based on Fejér kernels in [14].

The question of extremal functions to attain equality in Breitenberger's periodic uncertainty principle is not addressed in these papers. As it turns out that there is no function satisfying the equality, our goal is to construct asymptotically optimal functions. Not surprisingly, this leads to periodized Gaussian functions, i.e., special cases of Theta functions (see Section 2). Additionally, the wavelet functions in the corresponding multiresolution analysis also show a similar asymptotic behaviour (see Section 3). In Section 4, the localization properties of the orthogonalized functions are considered.

Finally, let us remark that recently, Narcowich and Ward [11, 12] also studied wavelets and localization properties on higher dimensional spheres, while asymptotically optimal results for compactly supported scaling functions and wavelets on the real line can be found in the papers by Chui and Wang [4-6]. Note also that an approach to optimal functions of an uncertainty principle for ultraspherical expansions has been studied recently by Rösler and Voit [15].

\section{Time frequency uncertainty}

Let $L_{2 \pi}^{2}$ denote the space of complex-valued square-integrable $2 \pi$-periodic functions with inner product $\langle f, g\rangle=\frac{1}{2 \pi} \int_{0}^{2 \pi} f(x) \overline{g(x)} d x$ and norm $\|f\|_{2}=\left(\frac{1}{2 \pi} \int_{0}^{2 \pi}|f(x)|^{2} d x\right)^{1 / 2}$. To state an uncertainty principle for functions in $L_{2 \pi}^{2}$, the necessary notions of variance for periodic functions need to be introduced. We refer to Breitenberger [3] for a discussion of the physics background and Narcowich and Ward [10] for applications to periodic basis functions in a multiresolution analysis.

Definition 2.1. For a function $f \in L_{2 \pi}^{2}$, represented by its Fourier series, i.e., $f(x)=\sum_{s=-\infty}^{\infty} c_{s} e^{i s x}$, the first trigonometric moment is defined as

$$
\tau(f):=\frac{1}{2 \pi} \int_{0}^{2 \pi} e^{i x}|f(x)|^{2} d x=\sum_{s=-\infty}^{\infty} c_{s} \overline{c_{s+1}} .
$$

Then, the angle variance for $f \in L_{2 \pi}^{2}$ is defined by

$$
\operatorname{var}_{A}(f):=\frac{\|f\|_{2}^{4}-|\tau(f)|^{2}}{|\tau(f)|^{2}}=\left|\frac{\sum_{s=-\infty}^{\infty}\left|c_{s}\right|^{2}}{\sum_{s=-\infty}^{\infty} c_{s} \bar{c}_{s+1}}\right|^{2}-1
$$

and the frequency variance for $f \in L_{2 \pi}^{2}$ as

$$
\operatorname{var}_{F}(f):=\left\|f^{\prime}\right\|_{2}^{2}+\frac{\left\langle f^{\prime}, f\right\rangle^{2}}{\|f\|_{2}^{2}}=\sum_{s=-\infty}^{\infty} s^{2}\left|c_{s}\right|^{2}-\frac{\left(\sum_{s=-\infty}^{\infty} s\left|c_{s}\right|^{2}\right)^{2}}{\sum_{s=-\infty}^{\infty}\left|c_{s}\right|^{2}}
$$


Note that the variances attain the value $\infty$ iff $\tau(f)=0$ or $f^{\prime}$ does not exist in $L_{2 \pi}^{2}$, respectively.

In the following theorem, an uncertainty relation for $L_{2 \pi}^{2}$ is formulated, which only excludes single frequency functions of the form $c e^{i k x}, c \in \mathbb{C}, k \in \mathbb{Z}$. In this case, $\operatorname{var}_{F}=0$ and $\operatorname{var}_{A}=\infty$. Nevertheless, inequality (2.1) below actually holds for all functions in $L_{2 \pi}^{2}$. In order to investigate the existence of optimal or asymptotically optimal functions for this uncertainty inequality, and for the sake of completeness, we give a detailed proof here.

Theorem 2.1. (Uncertainty Principle for periodic functions) $([3,10])$. For functions $f \in L_{2 \pi}^{2}$ which are not of the form $c e^{i k x}, c \in \mathbb{C}, k \in \mathbb{Z}$, it holds that

$$
\frac{\sqrt{\operatorname{var}_{A}(f) \operatorname{var}_{F}(f)}}{\|f\|_{2}} \geq \frac{1}{2} .
$$

Proof. The goal is to establish the inequality

$$
|\tau(f)|\|f\|_{2} \leq 2 \sqrt{\|f\|_{2}^{4}-|\tau(f)|^{2}} \sqrt{\left\|f^{\prime}\right\|_{2}^{2}\|f\|_{2}^{2}+\left\langle f^{\prime}, f\right\rangle^{2}} .
$$

Without loss of generality one can assume $\|f\|_{2}=1$, which reduces (2.1) to

$$
|\tau(f)| \leq 2 \sqrt{1-|\tau(f)|^{2}} \sqrt{\left\|f^{\prime}\right\|_{2}^{2}+\left(f^{\prime}, f\right)^{2}} .
$$

Setting for notional convenience $\tau=\tau(f) \in \mathbb{C}$, and $\mu=-i\left\langle f^{\prime}, f\right\rangle \in \mathbb{R}$, it holds that

$$
1-|\tau|^{2}=\left\|\left(e^{i \cdot}-\tau\right) f\right\|_{2}^{2}
$$

and

$$
\left\|f^{\prime}\right\|_{2}^{2}-\mu^{2}=\left\|-i f^{\prime}-\mu f\right\|_{2}^{2}
$$

One directly verifies the identity

$$
e^{i x} f(x)=-i\left[\left(e^{i x}-\tau\right) f\right]^{\prime}(x)-\left(e^{i x}-\tau\right)[-i f]^{\prime}(x)
$$

and, consequently,

$$
\begin{aligned}
e^{i x} f(x)= & \left\{-i\left[\left(e^{i \cdot}-\tau\right) f\right]^{\prime}(x)-\mu\left(e^{i x}-\tau\right) f(x)\right\} \\
& -\left\{\left(e^{i x}-\tau\right)(-\mu) f(x)+\left(e^{i x}-\tau\right)(-i) f^{\prime}(x)\right\}
\end{aligned}
$$


Taking inner products with $f$ yields

$$
\begin{aligned}
\tau & =\left\langle-i\left[\left(e^{i}-\tau\right) f\right]^{\prime}-\mu\left(e^{i}-\tau\right) f, f\right\rangle-\left\langle\left(e^{i}-\tau\right)(-\mu) f+\left(e^{i .}-\tau\right)(-i) f^{\prime}, f\right\rangle \\
& =: A_{1}+A_{2} .
\end{aligned}
$$

Using integration by parts $A_{1}$ produces

$$
A_{1}=\left\langle\left(e^{i \cdot}-\tau\right) f,-i f^{\prime}\right\rangle-\left\langle\mu\left(e^{i \cdot}-\tau\right) f, f\right\rangle=\left\langle\left(e^{i \cdot}-\tau\right) f,-i f^{\prime}-\mu f\right\rangle,
$$

and thus by the Cauchy-Schwarz inequality

$$
\left|A_{1}\right| \leq\left\|\left(e^{i \cdot}-\tau\right) f\right\|_{2}\left\|-i f^{\prime}-\mu f\right\|_{2} .
$$

For $A_{2}$, one obtains

$$
A_{2}=-\left\langle-i f^{\prime}-\mu f,\left(e^{-i \cdot}-\bar{\tau}\right) f\right\rangle
$$

and, once again by the Cauchy-Schwarz inequality,

$$
\left|A_{2}\right| \leq\left\|-i f^{\prime}-\mu f\right\|_{2}\left\|\left(e^{-i \cdot}-\bar{\tau}\right) f\right\|_{2} .
$$

Taking into account that $\left\|\left(e^{-i \cdot}-\bar{\tau}\right) f\right\|_{2}=\left\|\left(e^{i \cdot}-\tau\right) f\right\|_{2}$, inequality (2.2) follows from equations (2.3) and (2.4).

Now, we investigate the problem of optimal periodic time-frequency localization, i.e., whether it is possible to achieve equality in the uncertainty principle, which in turn implies equality in (2.1). Examining the proof, in the Cauchy-Schwarz inequality actual equality only occurs iff for $A_{1}$

$$
c_{1}\left(e^{i x}-\tau\right) f(x)=-i f^{\prime}(x)-\mu f(x) \text { - a.e., }
$$

and for $A_{2}$

$$
c_{2}\left(e^{-i x}-\bar{\tau}\right) f(x)=-i f^{\prime}(x)-\mu f(x) \quad \text { a.e. }
$$

with complex constants $c_{1}, c_{2}$.

Apart from the functions $c e^{i k x}$ mentioned above, where $-i f^{\prime}-\mu f \equiv 0$, this would only be possible if on a set of positive measure and for a constant $c \in \mathbb{C}$, we have

$$
e^{i x}-\tau=c\left(e^{-i x}-\bar{\tau}\right),
$$

i.e.,

$$
e^{i x}-c e^{-i x}=\tau-c \bar{\tau}
$$


which cannot happen as the left hand side is not constant. Hence, there is no function $f$ of optimal time-frequency localization. However, one can try to obtain the best possible constant 0.5 in the limit which will be the goal for the rest of this section.

Let us consider the Theta functions

$$
\theta(z, q)=\sum_{s=-\infty}^{\infty} q^{s^{2}} z^{s} \quad(z \in \mathbb{C} \backslash\{0\},|q|<1)
$$

With the transformation $z=e^{i x}, q=e^{-h}$, we write

$$
\varphi_{h}(x)=\theta\left(e^{i x}, e^{-h}\right)=\sum_{s=-\infty}^{\infty} e^{-h s^{2}} e^{i s x} .
$$

Note that $\varphi_{h}$ is an even positive $2 \pi$-periodic and smooth function.

Before we prove the essential theorems, we need some auxiliary results and estimates which are summarized in the following lemma.

Lemma 2.1. For all $h>0$, and shifts $\alpha \in \mathbb{R}$, the following identity holds

$$
\sum_{s=-\infty}^{\infty} e^{-2 h(s-\alpha)^{2}}=\sqrt{\frac{\pi}{2 h}} \sum_{s=-\infty}^{\infty} \cos 2 \pi s \alpha e^{-\frac{\pi^{2} 2}{2 h}}
$$

Consequently, the following estimates hold for the function

$$
u(h, \alpha):=\sum_{s=-\infty}^{\infty} e^{-2 h(s-\alpha)^{2}},
$$

independently of $\alpha$,

$$
\sqrt{\frac{\pi}{2 h}} \frac{1-3 e^{-\frac{2^{2}}{2 h}}}{1-e^{-\frac{2^{2}}{2 h}}}<u(h, \alpha)<\sqrt{\frac{\pi}{2 h}} \frac{1+e^{-\frac{x^{2}}{2 h}}}{1-e^{-\frac{x^{2}}{2 h}}},
$$

and more precisely for $u(h):=u(h, 0)$,

$$
\sqrt{\frac{\pi}{2 h}}<u(h)<\sqrt{\frac{\pi}{2 h}} \frac{1+e^{-\frac{\pi^{2}}{2 \hbar}}}{1-e^{-\frac{\pi^{2}}{2 h}}} .
$$

Proof. The Poisson summation formula (see also [2, Chap. 3]) can be used to establish (i) immediately. The sum $\sum_{s=-\infty}^{\infty} e^{-\frac{k_{2}^{2}}{2 h}}$ can be easily estimated as a geometric series 


$$
1<\sum_{s=-\infty}^{\infty} e^{-\frac{n^{2} 2}{2 \hbar}}<1+2 \sum_{s=1}^{\infty} e^{-\frac{\pi_{s}^{2}}{2 \hbar}}=\frac{1+e^{-\frac{\pi^{2}}{2 \hbar}}}{1-e^{-\frac{\pi^{2}}{2 \hbar}}}
$$

yielding (iii).

Estimating $\sum_{s=-\infty}^{\infty} \cos 2 \pi s \alpha e^{-\frac{\pi^{2} s^{2}}{2 h}}$ from below by $2-\sum_{s=-\infty}^{\infty} e^{-\frac{\pi^{2} p^{2}}{2 \hbar}}$, one finally obtains the lower bound in (ii).

It is now possible to give an estimate for the uncertainty product of the Theta functions $\varphi_{h}$.

Theorem 2.2. For all $0<h \leq 1$, it holds that

$$
\frac{\sqrt{\operatorname{var}_{A}\left(\varphi_{h}\right) \operatorname{var}_{F}\left(\varphi_{h}\right)}}{\left\|\varphi_{h}\right\|_{2}}<\frac{1+\sqrt{h}}{2}
$$

Proof. Using the Fourier coefficients $c_{s, h}=e^{-h s^{2}}$ of $\varphi_{h}$, we write

$$
\sum_{s=-\infty}^{\infty} c_{s, h}^{2}=\sum_{s=-\infty}^{\infty} e^{-2 h s^{2}}=u(h)
$$

and

$$
\begin{aligned}
\sum_{s=-\infty}^{\infty} c_{s, h} c_{s+1, h} & =\sum_{s=-\infty}^{\infty} e^{-h\left(s^{2}+(s+1)^{2}\right)}=e^{-\frac{h}{2}} \sum_{s=-\infty}^{\infty} e^{-\frac{h}{2}(2 s+1)^{2}} \\
& =e^{-\frac{h}{2}}\left(\sum_{s=-\infty}^{\infty} e^{-\frac{h}{2} s^{2}}-\sum_{s=-\infty}^{\infty} e^{-\frac{h}{2}(2 s)^{2}}\right)=e^{-\frac{h}{2}}\left(u\left(\frac{h}{4}\right)-u(h)\right)
\end{aligned}
$$

Hence we obtain

$$
\operatorname{var}_{A}\left(\varphi_{h}\right)=\frac{u^{2}(h)}{e^{-h}\left(u\left(\frac{h}{4}\right)-u(h)\right)^{2}}-1 .
$$

Now it follows by Lemma 2.1

$$
\begin{aligned}
u\left(\frac{h}{4}\right)-u(h) & >\sqrt{\frac{2 \pi}{h}}-\sqrt{\frac{\pi}{2 h}} \frac{1+e^{-\frac{\pi^{2}}{2 \hbar}}}{1-e^{-\frac{\pi^{2}}{2 h}}} \\
& =\sqrt{\frac{\pi}{2 h}}\left(\frac{1-3 e^{-\frac{\pi^{2}}{2 h}}}{1-e^{-\frac{\pi^{2}}{2 h}}}\right) .
\end{aligned}
$$


Then

$$
\begin{aligned}
\operatorname{var}_{A}\left(\varphi_{h}\right) & <e^{h}\left(\frac{1+e^{-\frac{\frac{2}{2}^{2}}{2}}}{1-3 e^{-\frac{\frac{2}{2}^{2}}{2 h}}}\right)^{2}-1 \\
& =e^{h}-1+8 e^{h}\left(\frac{1}{e^{\frac{\frac{2}{2}^{2}}{2}}-3}+\frac{2}{\left(e^{\frac{\frac{1}{2}^{2}}{2 h}}-3\right)^{2}}\right)
\end{aligned}
$$

Taylor's formula and the monotonicity of $h^{2}\left(e^{\frac{x^{2}}{2 h}}-3\right)$ for $0<h \leq 1$ give the estimate

$$
\operatorname{var}_{A}\left(\varphi_{h}\right)<h+\frac{e h^{2}}{2}+8 e\left(\frac{h^{2}}{e^{\frac{x^{2}}{2}}-3}+\frac{2 h^{2}}{\left(e^{\frac{z^{2}}{2}}-3\right)^{2}}\right)<h+1.53 h^{2}
$$

Now we have to deal with $\operatorname{var}_{F}\left(\varphi_{h}\right)$. Differentiation of the Poisson summation formula with respect to $h$ yields

$$
\begin{aligned}
\operatorname{var}_{F}\left(\varphi_{h}\right) & =\sum_{s=-\infty}^{\infty} s^{2} e^{-2 h s^{2}}=-\frac{1}{2} \frac{d}{d h}\left(\sum_{s=-\infty}^{\infty} e^{-2 h s^{2}}\right) \\
& =-\frac{1}{2} \frac{d}{d h}\left(\sqrt{\frac{\pi}{2 h}} \sum_{s=-\infty}^{\infty} e^{-\frac{k^{2} 2^{2}}{2 h}}\right) \\
& =\frac{1}{4 h} \sqrt{\frac{\pi}{2 h}} \sum_{s=-\infty}^{\infty} e^{-\frac{\pi^{2} s^{2}}{2 h}}-\sqrt{\frac{\pi}{2 h}} \frac{\pi^{2}}{4 h^{2}} \sum_{s=-\infty}^{\infty} s^{2} e^{-\frac{\pi^{2} r^{2}}{2 h}} \\
& <\frac{1}{4 h} \sqrt{\frac{\pi}{2 h}} \sum_{s=-\infty}^{\infty} e^{-\frac{k^{2}}{2 h}}=\frac{u(h)}{4 h},
\end{aligned}
$$

where the last equality follows again from Lemma 2.1 .

Putting together the corresponding estimates we obtain

$$
\frac{\operatorname{var}_{A}\left(\varphi_{h}\right) \operatorname{var}_{F}\left(\varphi_{h}\right)}{\left\|\varphi_{h}\right\|_{2}^{2}}<\frac{\left(h+1.53 h^{2}\right) u(h)}{u(h) 4 h}<\frac{1}{4}+0.39 h,
$$

from which the assertion of Theorem 2.1 follows.

Note that $h=0$ yields a distribution, but not an $L_{2 \pi}^{2}$-function. As an alternative, one can investigate the limit case $h=0$ for the partial sum $\sum_{s=-m}^{m} e^{-h s^{2}} e^{i s x}$ of $\varphi_{h}$, i.e.,

$$
\phi_{m}(x)=\sum_{s=-m}^{m} e^{i s x}
$$


One obtains

$$
\begin{aligned}
\frac{\sqrt{\operatorname{var}_{A}\left(\phi_{m}\right) \operatorname{var}_{F}\left(\phi_{m}\right)}}{\left\|\phi_{m}\right\|_{2}} & =\sqrt{\frac{\frac{(2 m+1)^{2}-(2 m)^{2}}{(2 m)^{2}} \sum_{s=-m}^{m} s^{2}}{2 m+1}} \\
& =\sqrt{\frac{(4 m+1) m(m+1)(2 m+1)}{4 m^{2}(2 m+1) 3}} \\
& =\sqrt{\frac{(4 m+1)(m+1)}{12 m}} .
\end{aligned}
$$

Hence,

$$
\sqrt{\frac{m}{3}+\frac{5}{12}}<\frac{\sqrt{\operatorname{var}_{A}\left(\phi_{m}\right) \operatorname{var}_{F}\left(\phi_{m}\right)}}{\left\|\phi_{m}\right\|_{2}}<\sqrt{\frac{m}{3}+\frac{1}{2}}
$$

For similar results, see $[10,14,16]$. However, in [14], it is proved that a certain smoothing procedure, namely a Fejér mean of the partial sum polynomial $\phi_{m}$, i.e.,

$$
\sigma_{m}=\frac{1}{m+1} \sum_{k=0}^{m} \phi_{k}
$$

yields

$$
\frac{\sqrt{\operatorname{var}_{A}\left(\sigma_{m}\right) \operatorname{var}_{F}\left(\sigma_{m}\right)}}{\left\|\sigma_{m}\right\|_{2}}=\sqrt{\frac{3}{-10}\left(1+\frac{20 m^{2}+1}{2\left(8 m^{2}+1\right)\left(4 m^{2}-1\right)}\right)}
$$

which in the limit $m \rightarrow \infty$ tends to $\sqrt{0.3}=0.5477 \ldots$

At this point let us mention that the functions $\varphi_{h}$ of the type (2.5) are clearly not the only ones which yield asymptotically optimal uncertainty products. As other important examples we refer to trigonometric polynomials where the Fourier coefficients are values of $B$-splines. In connection with the central limit theorem it is shown in [17, Theorem 1] that certain scaled versions of $B$-splines converge pointwise and in $L^{p}$-norms, with $p \geq 2$, to the Gaussian (or its scaled version). So one obtains trigonometric polynomials which have an uncertainty product less than $(1+\epsilon) / 2$ for an appropriate scaling and a big enough order $d$ of the spline. Our functions $\phi_{m}$ and $\sigma_{m}$ are related to the order $d=0$ and $d=1$.

To illustrate these results, see Figure 1 for Theta functions with two different step sizes, and Figure 2 for a Dirichlet and a Fejér kernel. 

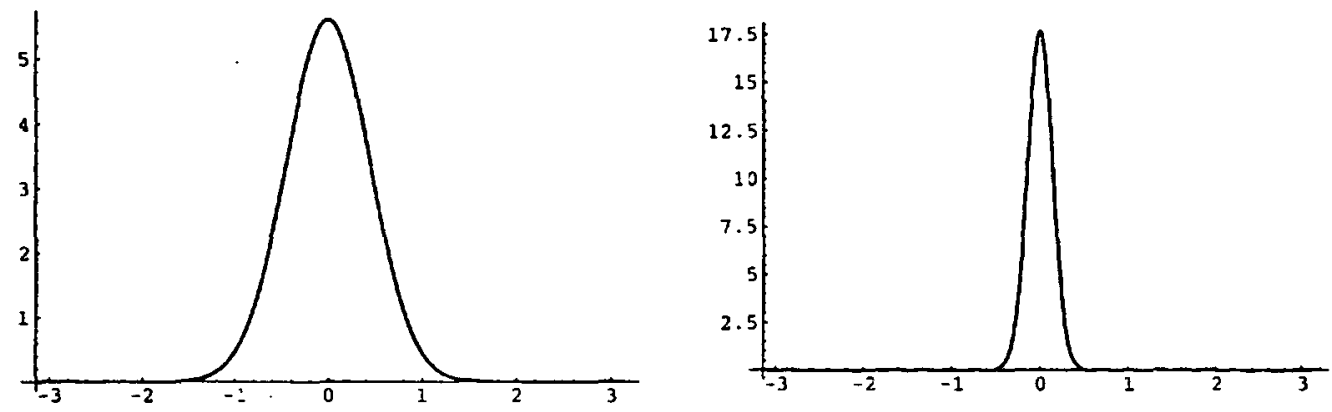

FIGURE 1: $\varphi_{0.1}$ (left) and $\varphi_{0.01}$ (right):
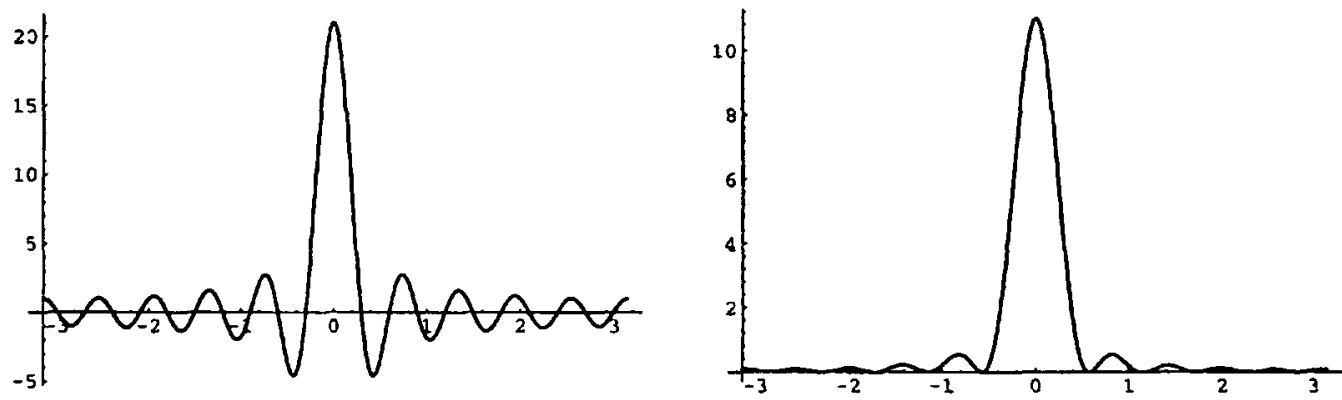

FIGURE 2: $\phi_{10}$ (left) and $\sigma_{10}$ (right).

\section{Multiresolution analysis}

Now, the goal is to construct nested spaces spanned by translates of the Theta functions investigated in Section 2. For fixed $h$, we set

$$
V_{n}=\operatorname{span}\left\{\varphi_{h}\left(.-\frac{2 k \pi}{n}\right), k=0, \ldots, n-1\right\} .
$$

Trivially, it holds that

$$
V_{n} \subset V_{2 n}
$$

Furthermore, as all the Fourier coefficients of the function $\varphi_{h}$ are positive, we have

$$
\operatorname{dim} V_{n}=n \text { and } L_{2 \pi}^{2}=\cos _{L^{2}}\left(\bigcup_{j=0}^{\infty} V_{2^{\prime}}\right)
$$

See, for example, Lemma 2.4 (i) and Theorem 3.1 in [13]. 
Hence, it makes sense to consider wavelet spaces $W_{n}$, namely the relative orthogonal complements of $V_{n}$ with respect to $V_{2 n}$ such that

$$
V_{2 n}=V_{n} \oplus W_{n}
$$

While the scaling functions are just translates of one single function, independent of the level $n$, the corresponding wavelets turn out to be dependent on $n$. As the major concern here, however, is the dependence on the term $h$, we will suppress the dependence on $n$ in our notation by writing

$$
\psi_{h}(x):=\sum_{s=-\infty}^{\infty} e^{-h s^{2}}\left(1-\frac{\sum_{p=-\infty}^{\infty}(-1)^{p} e^{-2 h(n p-s)^{2}}}{\sum_{q=-\infty}^{\infty} e^{-2 h(n q-s)^{2}}}\right) e^{i s\left(x-\frac{n}{n}\right)}
$$

One now obtains

Theorem 3.1. It holds for $n \in \mathbb{N}$ that

$$
W_{n}=\operatorname{span}\left\{\psi_{h}\left(-\frac{2 k \pi}{n}\right), k=0, \ldots, n-1\right\}
$$

Proof. The fact that $\psi_{h}$ is indeed an element of $V_{2 n}$ follows immediately from the $2 n$-periodicity in $s$ of the quotient

$$
\frac{d_{s, h}}{c_{s, h}}=\left(1-\frac{\sum_{p=-\infty}^{\infty}(-1)^{p} e^{-2 h(n p-s)^{2}}}{\sum_{q=-\infty}^{\infty} e^{-2 h(n q-s)^{2}}}\right) e^{-i s \frac{x}{n}}
$$

where $d_{s, h}$ are the Fourier coefficients of $\psi_{h}$, see Lemma 2.1 of [13]. The orthogonality assertion is equivalent to

$$
\sum_{s=-\infty}^{\infty} c_{s, h} d_{s, h} e^{2 \pi i{ }_{n}}=0, \quad r=0, \ldots, n-1
$$

Hence we have to prove

$$
\sum_{s=-\infty}^{\infty} e^{-2 h s^{2}} e^{\pi i s \frac{2 r-1}{n}}=\sum_{s=-\infty}^{\infty} e^{-2 h s^{2}} e^{\pi i s \frac{2 r-1}{n}} \frac{\sum_{p=-\infty}^{\infty}(-1)^{p} e^{-2 h(n p-s)^{2}}}{\sum_{q=-\infty}^{\infty} e^{-2 h(n q-s)^{2}}}
$$

Setting $s=n \ell+t$ yields 


$$
\sum_{s=-\infty}^{\infty}=\sum_{i=0}^{n-1} \sum_{\ell=-\infty}^{\infty}
$$

and we obtain for the right hand side

$$
\begin{aligned}
& \sum_{t=0}^{n-1} \sum_{\ell=-\infty}^{\infty} e^{-2 h(n \ell+t)^{2}}(-1)^{\ell} e^{\pi i t \frac{2 r-1}{n}} \frac{\sum_{p=-\infty}^{\infty}(-1)^{p} e^{-2 h(n(p-\ell)-t)^{2}}}{\sum_{q=-\infty}^{\infty} e^{-2 h(n(q-\ell)-t)^{2}}} \\
& =\sum_{t=0}^{n-1} e^{\pi i t \frac{2 r-1}{n}} \sum_{p=-\infty}^{\infty}(-1)^{p} e^{-2 h(n p-t)^{2}}
\end{aligned}
$$

which equals the left hand side.

Additionally, one can also state the two-scale relations for the basis functions of $V_{n}$ and $W_{n}$ with respect to $V_{2 n}$. We have

Corollary 3.1. For fixed $n \in \mathbb{N}$, and $k=0, \ldots, n-1$, the two-scale relations are

$$
\begin{aligned}
& \varphi_{h}\left(x-\frac{2 k \pi}{n}\right)=\sum_{\ell=0}^{2 n-1} \alpha_{k, \ell} \varphi_{h}\left(x-\frac{\ell \pi}{n}\right) \\
& \psi_{h}\left(x-\frac{2 k \pi}{n}\right)=\sum_{\ell=0}^{2 n-1} \beta_{k, \ell} \varphi_{h}\left(x-\frac{\ell \pi}{n}\right)
\end{aligned}
$$

where for $m=0, \ldots, n-1$,

$$
\begin{aligned}
& \beta_{k, 2 m+1}=\delta_{k, m}, \\
& \beta_{k, 2 m}=\frac{1}{n} \sum_{t=0}^{n-1} e^{-\pi i t \frac{2 m-2 k-1}{n}} \frac{\sum_{p=-\infty}^{\infty}(-1)^{p} e^{-2 h(n p-t)^{2}}}{\sum_{q=-\infty}^{\infty} e^{-2 h(n q-t)^{2}}},
\end{aligned}
$$

and, trivially, $\alpha_{k, \ell}=\delta_{2 k, \ell}$.

Proof. The values of the $\beta_{k, \ell}$ 's are obtained by applying discrete Fourier transforms to the corresponding two-scale equation, see also [13].

Now, the question of time-frequency localization of the wavelet will be addressed.

Theorem 3.2. Let $n \in \mathbb{N}$ be fixed. Then, for all $0<h \leq \frac{1}{4 n^{2}}$, we have

$$
\frac{\sqrt{\operatorname{var}_{A}\left(\psi_{h}\right) \operatorname{var}_{F}\left(\psi_{h}\right)}}{\left\|\psi_{h}\right\|_{2}}<\frac{1}{2}+1.1 n^{2} \sqrt{h} .
$$


Proof. As the variance measures of Definition 2.1 are invariant under translation of the function, we consider $\tilde{\psi}_{h}=\psi_{h}\left(\cdot+\frac{\pi}{n}\right)$ with Fourier coefficients

$$
\tilde{d}_{s, h}=c_{s, h}\left(1-\frac{\sum_{p=-\infty}^{\infty}(-1)^{p} e^{-2 h(n p-s)^{2}}}{\sum_{q=-\infty}^{\infty} e^{-2 h(n q-s)^{2}}}\right) .
$$

First, we obtain

$$
\begin{aligned}
\frac{\sum_{p=-\infty}^{\infty}(-1)^{p} e^{-2 h(n p-s)^{2}}}{\sum_{q=-\infty}^{\infty} e^{-2 h(n q-s)^{2}}} & =\frac{\sum_{p=-\infty}^{\infty} e^{-2 h(2 p n-s)^{2}}-\sum_{p=-\infty}^{\infty} e^{-2 h(2 p n+n-s)^{2}}}{u\left(n^{2} h, \frac{s}{n}\right)} \\
& =\frac{u\left(4 n^{2} h, \frac{s}{2 n}\right)-u\left(4 n^{2} h, \frac{s}{2 n}-\frac{1}{2}\right)}{u\left(n^{2} h, \frac{s}{n}\right)} .
\end{aligned}
$$

Hence, by Lemma 2.1, with $\gamma=\frac{\pi^{2}}{8 h n^{2}}$,

$$
\begin{aligned}
\left|\frac{\sum_{p=-\infty}^{\infty}(-1)^{p} e^{-2 h(n p-s)^{2}}}{\sum_{q=-\infty}^{\infty} e^{-2 h(n q-s)^{2}}}\right| & <\frac{1}{2}\left(\frac{1+e^{-\gamma}}{1-e^{-\gamma}}-\frac{1-3 e^{-\gamma}}{1-e^{-\gamma}}\right) \frac{1-e^{-4 \gamma}}{1-3 e^{-4 \gamma}} \\
& =\frac{1}{2} \frac{4 e^{-\gamma}}{1-e^{-\gamma}} \frac{1-e^{-4 y}}{1-3 e^{-4 \gamma}} .
\end{aligned}
$$

Consequently,

$$
\left|\frac{\sum_{p=-\infty}^{\infty}(-1)^{p} e^{-2 h(n p-s)^{2}}}{\sum_{q=-\infty}^{\infty} e^{-2 h(n q-s)^{2}}}\right|<2.02 e^{-\frac{x^{2}}{8 h n^{2}}}
$$

and

$$
\left(1-2.02 e^{-\gamma}\right) c_{s, h} \leq \tilde{d}_{s, h} \leq\left(1+2.02 e^{-\gamma}\right) c_{s, h}
$$

In the following, we will use that

$$
\frac{1+2.02 e^{-\gamma}}{1-2.02 e^{-\gamma}}<1+4.1 e^{-\gamma}<1+0.06 \sqrt{h n}
$$

and

$$
\left(\frac{1+2.02 e^{-\gamma}}{1-2.02 e^{-\gamma}}\right)^{4}<1+17.2 e^{-\gamma}<1+1.98 h^{2} n^{4}
$$


According to Section 2, we consider

$$
\frac{\sqrt{\operatorname{var}_{A}\left(\psi_{h}\right) \operatorname{var}_{F}\left(\psi_{h}\right)}}{\left\|\psi_{h}\right\|_{2}}=\sqrt{-1+\left(\frac{\sum_{s=-\infty}^{\infty} \tilde{d}_{s, h}^{2}}{\sum_{s=-\infty}^{\infty} \tilde{d}_{s, h} \tilde{d}_{s+1, h}}\right)^{2}} \sqrt{\frac{\sum_{s=-\infty}^{\infty} s^{2} \tilde{d}_{s, h}^{2}}{\sum_{s=-\infty}^{\infty} \tilde{d}_{s, h}^{2}}}
$$

and obtain as an upper bound for the first squareroot term

$$
R_{1}=\sqrt{-1+\left(1+17.2 e^{-\gamma}\right)\left(\frac{\sum_{s=-\infty}^{\infty} c_{s, h}^{2}}{\sum_{s=-\infty}^{\infty} c_{s, h} c_{s+1, h}}\right)^{2}}
$$

and for the second one

$$
R_{2}=\sqrt{\left(1+4.1 e^{-\gamma}\right)^{2} \frac{\sum_{s=-\infty}^{\infty} s^{2} c_{s, h}^{2}}{\sum_{s=-\infty}^{\infty} c_{s, h}^{2}}}
$$

Using the corresponding estimates in the proof of Theorem 2.2, we obtain the desired result.

Let us note here that the above proof also shows that the additional term $1.1 n^{2} \sqrt{h}$ is best possible up to the constant.

It remains an open question whether there is a translation invariant basis of $W_{n}$ which is asymptotically optimal independent of the level $n$. More generally, we do not know any orthogonal decomposition of $L_{2 \pi}^{2}$ in wavelet spaces where every wavelet has an asymptotically optimal uncertainty product. However, an example of an orthogonal basis with uniformly bounded uncertainty product can be found in [16].

Figure 3 illustrates the influence of different values for the parameter $h$ and the level $n$. For $n=1$ there is only one scaling function $\varphi_{h}$ (dashed line) and one wavelet $\psi_{h}$ (thick line). For $n=4$ and $n=8$, we show two scaling functions $\varphi_{h}, \varphi_{h}\left(\cdot-\frac{2 \pi}{n}\right)$ and the wavelet $\psi_{h}$.

\section{Orthogonalization}

Finally, we will consider time-frequency localization for orthogonal scaling functions and wavelets.

Lemma 4.1. For fixed $h$, let

$$
\varphi_{h}^{\perp}(x):=\sum_{s=-\infty}^{\infty} c_{s, h}^{\perp} e^{i s x}
$$

with 


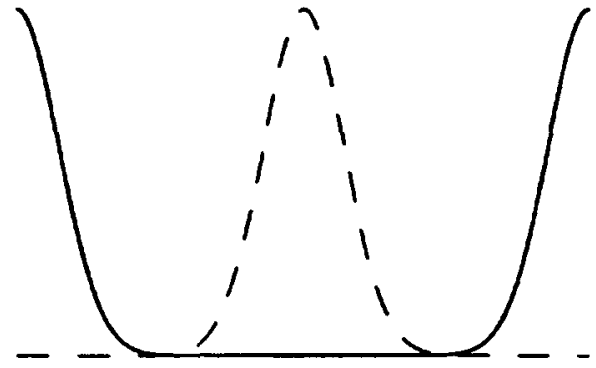

$h=0.1$ and $n=1$

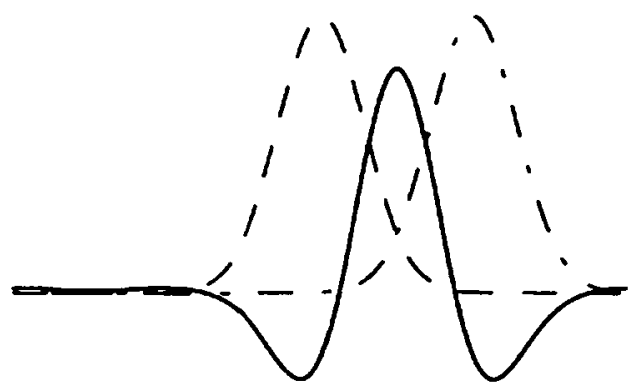

$h=0.1$ and $n=4$

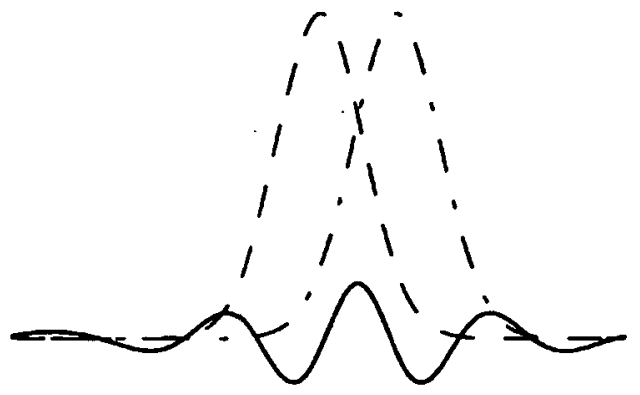

$h=0.1$ and $n=8$
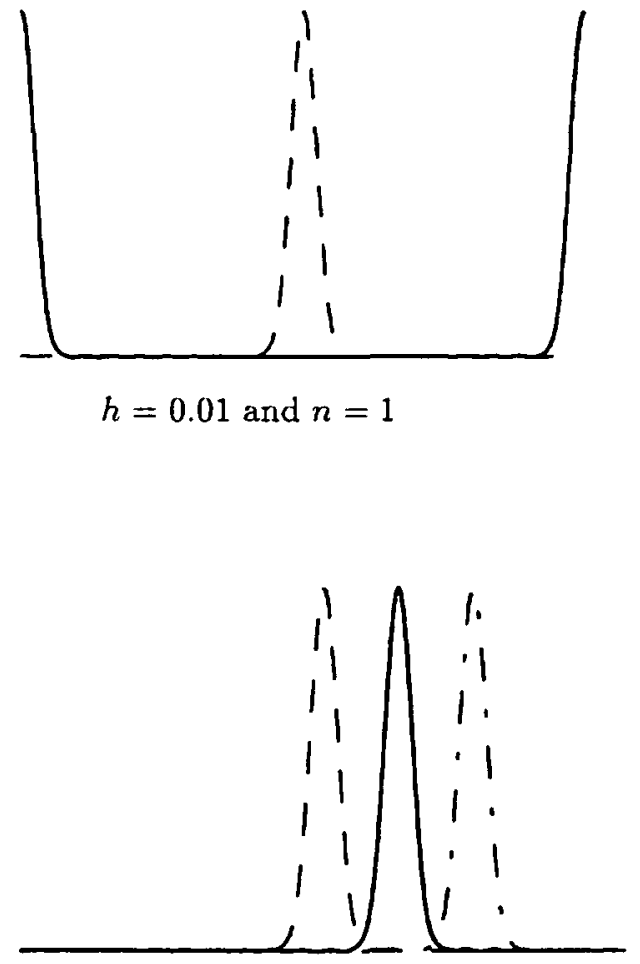

$h=0.01$ and $n=4$

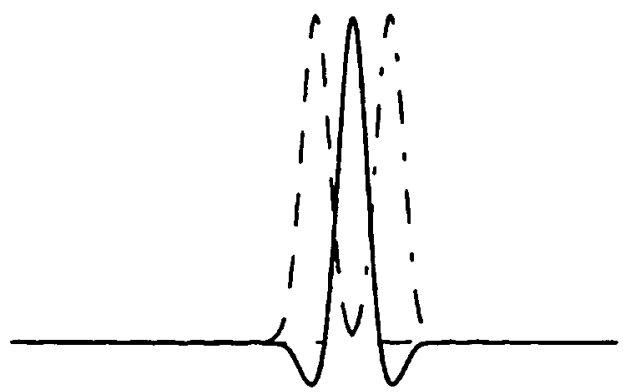

$h=0.01$ and $n=8$

FIGURE 3 


$$
c_{s, h}^{\perp}:=e^{-h s^{2}}\left(n \sum_{q=-\infty}^{\infty} e^{-2 h(s+q n)^{2}}\right)^{-\frac{1}{2}}
$$

and

$$
\psi_{h}^{\perp}(x):=\sum_{s=-\infty}^{\infty} d_{s, h}^{\perp} e^{i s\left(x-\frac{\pi}{n}\right)}
$$

with

$$
d_{s, h}^{\perp}:=e^{-h s^{2}}\left(\left(n \sum_{p=-\infty}^{\infty} e^{-2 h(s+2 p n)^{2}}\right)^{-1}-\left(n \sum_{q=-\infty}^{\infty} e^{-2 h(s+q n)^{2}}\right)^{-1}\right)^{\frac{1}{2}}
$$

Then $\varphi_{h}^{\frac{1}{2}}\left(\cdot-\frac{2 k \pi}{n}\right), \quad k=0, \ldots, n-1, \quad$ and $\quad \psi_{h}^{\perp}\left(\cdot-\frac{2 k \pi}{n}\right), \quad k=0, \ldots, n-1, \quad$ constitute orthonormal bases for $V_{n}$ and $W_{n}$, respectively.

Proof. We have to prove $\varphi_{h}^{\perp} \in V_{n}, \psi_{h}^{\perp} \in W_{n}$ and the orthonormality of corresponding translates. This can be done in exactly the same manner as in the proof of Theorem 3.1 (see also [13]).

Now we can show that for fixed $n$, these orthonormalized scaling functions and wavelets are again optimally time-frequency localized.

Theorem 4.1. Let $n$ be fixed. Then, for all $0<h \leq \frac{1}{4 n^{2}}$, we have

$$
\sqrt{\operatorname{var}_{A}\left(\varphi_{h}^{1}\right) \operatorname{var}_{F}\left(\varphi_{h}^{1}\right)}<\frac{1}{2}+n^{2} \sqrt{h}
$$

and

$$
\sqrt{\operatorname{var}_{A}\left(\psi_{h}^{\perp}\right) \operatorname{var}_{F}\left(\psi_{h}^{\perp}\right)}<\frac{1}{2}+1.1 n^{2} \sqrt{h}
$$

Proof. Because of the similarity to the proof of Theorem 3.2, we can be very brief here. Note that

$$
c_{s, h}^{\perp}=c_{s, h}\left(\frac{2 h}{\pi}\right)^{\frac{1}{4}}\left(u\left(h n^{2}, \frac{s}{n}\right)\right)^{-\frac{1}{2}}\left(\frac{2 h n^{2}}{\pi}\right)^{-\frac{1}{4}}
$$

With Lemma 2.1 and $\beta=\frac{\pi^{2}}{2 h n^{2}}>2 \pi^{2}$, we estimate 


$$
\begin{gathered}
\left|\left(u\left(h n^{2}, \frac{s}{n}\right)\right)^{-\frac{1}{2}}\left(\frac{2 h n^{2}}{\pi}\right)^{-\frac{1}{4}}-1\right| \\
<\max \left\{1-\left(\frac{1-e^{-\beta}}{1+e^{-\beta}}\right)^{\frac{1}{2}} ;\left(\frac{1-e^{-\beta}}{1-3 e^{-\beta}}\right)^{\frac{1}{2}}-1\right\}<1.01 e^{-\beta},
\end{gathered}
$$

which is similar to (3.1). Analogously,

$$
d_{s, h}^{\perp}=c_{s, h}\left(\frac{2 h}{\pi}\right)^{\frac{1}{4}}\left(\frac{1}{u\left(4 h n^{2}, \frac{s}{2 n}\right)}-\frac{1}{u\left(h n^{2}, \frac{s}{n}\right)}\right)^{\frac{1}{2}}\left(\frac{2 h n^{2}}{\pi}\right)^{-\frac{1}{4}}
$$

Here we estimate with $\gamma=\frac{\pi^{2}}{8 h n^{2}}>\frac{\pi^{2}}{2}$

$$
\begin{aligned}
& \left|\left(\frac{1}{u\left(4 h n^{2}, \frac{s}{2 n}\right)}-\frac{1}{u\left(h n^{2}, \frac{s}{n}\right)}\right)^{\frac{1}{2}}\left(\frac{2 h n^{2}}{\pi}\right)^{-\frac{1}{4}}-1\right| \\
& <\max \left\{1-\left(2 \frac{1-e^{-\gamma}}{1+e^{-\gamma}}-\frac{1-e^{-4 \gamma}}{1-3 e^{-4 \gamma}}\right)^{\frac{1}{2}} ;\left(2 \frac{1-e^{-\gamma}}{1-3 e^{-\gamma}}-\frac{1-e^{-4 \gamma}}{1+e^{-4 \gamma}}\right)^{\frac{1}{2}}-1\right\} \\
& <2.03 e^{-\gamma} .
\end{aligned}
$$

With (4.1) and (4.2), we complete the estimates as in the proof of Theorem 3.2.

\section{Conclusion}

For Breitenberger's periodic uncertainty principle of Theorem 2.1, we have shown that the lower bound 0.5 cannot be attained. On the other hand, the bound is optimal as we have constructed functions $\varphi_{h}$, whose uncertainty products, according to Theorem 2.2, are arbitrarily close to 0.5 . Using equally spaced translates, these functions $\varphi_{h}$ give rise to a multiresolution analysis. In Theorem 3.1 we construct corresponding wavelet functions which are again asymptotically optimal with respect to $h$. However, the localization of the wavelet is estimated only in dependence on the level $n$. This means that for fixed $h$ the localization is lost for large values of $n$. Nevertheless, for fixed $n$ and small $h$, the shape of the wavelets approaches the shape of the Theta functions. Analogous results are stated in Section 4 for orthogonalized bases. It remains an open question whether there is an orthogonal basis of $L_{2 \pi}^{2}$, which is asymptotically optimal independent from the level $n$.

Acknowledgements. The authors thank Dr. F. J. Narcowich for several stimulating discussions concerning the uncertainty principle for periodic functions and especially for pointing out the proof of Theorem 2.1. 


\section{REFERENCES}

1. J. Benedetto, C. Heil and D. WaLNUT, Uncertainty principles for time-frequency operators, in Operator Theory: Advances and Applications, Vol. 58 (Birkhäuser Verlag, Basel, 1992), $1-25$.

2. L. BERG, Asymptotische Darstellungen und Entwicklungen (Deutscher Verlag der Wissenschaften, Berlin, 1968).

3. E. Breitengerger, Uncertainty measures and uncertainty relations for angle observables, Found. Phys. 15 (1985), 353-364.

4. C. K. ChUi and J. Z. WANG, A study of compactly supported scaling functions and wavelets, in Wavelets, Images and Surface Fitting (P. J. Laurent, A. Le Méhauté and L. L. Schumaker (eds.), AKPeters, Boston, 1994), 121-140.

5. C. K. ChUI and J. Z. WANG, High-order orthonormal scaling functions and wavelets give poor time-frequency localization, J. Fourier Anal. Appl. 2 (1996), 415-426.

6. C. K. ChUI and J. Z. WANG, A study of asymptotically optimal time-frequency localization by scaling functions and wavelets (CAT Report \# 323, Texas A\&M University, 1996).

7. L. COHEN, Time-frequency distributions - a review, Proc. IEEE 77 (1989), 941-981.

8. I. Daubechies, Ten lectures on wavelets (CBMS-NSF Series in Appl. Math., SIAM, Philadelphia, 1992).

9. Y. W. Кон, S. L. Lee and H. H. TaN, Periodic orthogonal splines and wavelets, Appl. Comput. Harmonic Anal. 2 (1995), 201-218.

10. F. J. Narcowich and J. D. WARD, Wavelets associated with periodic basis functions, Appl. Comput. Harmonic Anal. 3 (1996), 40-56.

11. F. J. NARCowich and J. D. WARD, Non-stationary spherical wavelets for scattered data, in Approximation Theory VIII, Vol. 2: Wavelets and Multilevel Approximation (C. K. Chui and L. L. Schumaker (eds.), World Scientific, 1995), 301-308.

12. F. J. NARCowiCh and J. D. WARD, Non-stationary wavelets on the $m$-sphere for scattered data, Appl. Comput. Harmonic Anal. 3 (1996), 324-336.

13. G. PlonkA and M. TASCHE, A unified approach to periodic wavelets, in Wavelets: theory, algorithms, and applications (C. K. Chui, L. Montefusco, and L. Puccio (eds.), Academic Press, New York, 1994), 137-151.

14. J. Prestin and E. Quak, Time frequency localization of trigonometric Hermite operators, in Approximation Theory VIII, Vol. 2: Wavelets and Multilevel Approximation (C. K. Chui and L. L. Schumaker (eds.), World Scientific, 1995), 343-350.

15. M. RösLeR and M. VoIt, An uncertainty principle for ultraspherical expansions, J. Math. Anal. Appl. 209 (1997), 624-634.

16. K. Selig, Trigonometric wavelets and the uncertainty principle, in Approximation Theory (M. W. Müller, M. Felten and D. H. Mache (eds.), Math. Research, Vol. 86, Akademie Verlag, Berlin, 1995), 293-304. 
17. M. Unser, A. Aldroubi and M. Eden, On the asymptotic convergence of $B$-spline wavelets to Gabor functions, IEEE Trans. Inf. Theory 38 (1992), 864-872.

INSTITUTE OF BIOMATHEMATICS AND BIOMETRY GSF - National Research CENTER for

ENVIRONMENT AND HEALTH

85764 NEUHERBERG

GERMANY

E-mail address: prestin@gsf.de
SINTEF APPLIED Mathematics

POSTBOKS 124 BLINDERN

N-0314 OsLo

NORWAY

E-mail address: Ewald.Quak@math.sintef.no 